\title{
Discrete Event Simulation of Hospital Sterilization Logistics
}

\author{
Bojan RUPNIK, Radivoj NARDIN, Tomaž KRAMBERGER
}

\begin{abstract}
This paper deals with the optimization of the flow of sterile Reusable Medical Devices (RMD) in a general hospital which takes place between the centralized sterilization department and the operating theatres, clinics, hospital departments or ER. This paper addresses the problem as a logistics optimization problem that has to be solved when redesigning processes to improve material availability and reduce cost. To address the problem, the Discrete Event Simulation (DES) method was used. The results of the simulation show that the studied problem can be solved by two different strategies. The first strategy is an acquisition of additional surgical instrument, while the second one is careful planning and automated surgery scheduling.
\end{abstract}

Keywords: discrete event simulation; hospital logistics; sterilization process

\section{INTRODUCTION}

Health care spending has increased dramatically during the last few decades. Globally, health care spending increased faster than the economic growth. Based on a recent report, the countries spending the most on health care today allocate between $8.9 \%$ and $16.4 \%$ of their total gross domestic product (GDP) to health care costs [1].

Since the health care has become an increasingly heavy burden on national and individual budgets many developed countries are implementing policies to cut cost [2]. Different authors claim that the cost effectiveness of health care can be improved by up to 10 to $20 \%$ [3]. The annual report on hospitals in The Netherlands [4] identifies opportunities for improvement in logistics of goods and pharmaceuticals of 1 billion euros, and another 2 billion euros in patient logistics. Most of the reductions can be achieved by adopting uniform work processes, using standardized materials, optimizing the process of transport, disinfection and sterilization. Adequate planning and appropriate use of information technology are valuable improvement opportunities [2].

In order to achieve those goals, hospitals are currently under pressure to become more effective. Consequently, attention for optimizing the logistic processes involved in sterilization logistics, focused on the central sterilization department, has increased. The flow of reusable medical devices (RMD) which have to be sterilized is one of the secondary processes which affects the overall hospital performance at most. In this paper, we explore opportunities for improvements of the material flow of RMDs. The improvements are based on an aligned combination of improvements in work processes and information technology.

Several authors have adressed this problem in the last decade. For instance Reymondon and co-authors [5] propose a methodology enabling new grouping choices of RMDs into packages. It exploits an innovative sharing strategy, with the final goal to optimize the objective function corresponding to process and storage costs by reducing storage costs. In their strategy RMDs are shared between different types of surgical cases and are not dedicated to one specific type of case. The main goal of the study was to develop a grouping choice called hybrid solution, which is an alternative solution between the two extreme solutions: "One package for one surgical case" and "one package for one RMD", which do not enable to optimize our objective function. The idea is to find the hybrid solution of RMD grouping into packages, which optimizes an objective function composed of storage costs and process costs. The methodology includes two stages. In stage one, the first hybrid solution is built identifying the RMD with a sharing interest. The stage two leads to look for a better solution starting from the hybrid grouping choice found in stage one.

Tlahig and co-authors [6] deal with the problem of sterilization service configuration within a hospital network. Two alternatives are considered: in-house sterilization service for each hospital or central sterilization service (CSS) ensures this function for all hospitals in the network. CSS could lead to better resource utilization and considerable cost savings. However, CSS can be considered only if the hospitals in the network are located in the geographically limited area. Otherwise the CSS can cause additional unnecessary costs. Moreover, CSS increases the risk of sterile items unavailability. That is why adoption of CSS requires a high level of management to ensure the coordination and the satisfaction of all the network actors.

The problem of the centralization vs. decentralization of the sterilization service within a hospital, where each surgical service has its own sterilization department was addressed by Tlahig in the paper [7]. A two-stepped iterative approach solution was proposed. The first step consisted of finding the best configuration between the centralization and decentralization of the various sterilization service departments; in the second step they aimed to find the optimal size for the configuration achieved in the first step. The authors claim the idea of reducing in-house sterilization cost by optimizing sterilization logistics and the composition of the sets of sterile items is the best solution.

In the paper [2] van de Klundert and co-authors deal with the optimization of the flow of sterile instruments between the sterilization department and the operating theatre. The topic is especially of interest in view of the current attempts of hospitals to cut cost by outsourcing sterilization tasks. This paper discusses the optimization problems that have to be solved when redesigning processes to improve material availability and reduce cost. The improvements are based on an aligned combination of 
improvements in work processes and information technology.

Ozturk and co-authors [8] deal with the problem of minimizing the make span of washing operations in hospitals sterilization services. They consider a batchscheduling problem where RMD sets may have different sizes and different release dates for washing. In case all release dates are equal, the problem is reduced to a binpacking problem. A mixed integer linear programming model was used to minimize the make span of washing operations.

In general, the demand for sterile instruments is determined by the surgeries taking place. Some of the surgeries are planned, others may be emergencies. Most hospitals enter information on planned operations into the Hospital Information System (HIS). However, many hospitals do not provide information on the whereabouts of sterile instruments during the day, and hence cannot consider it when planning and executing the sterilization activities. At present, RFID technology [9] promises to make real time information on instruments available, but some difficulties remain to be unsolved. Moreover, hardware alone will not solve the problem. Software and organization, as needed to make effective use of relevant HIS functionality, are challenges as well. Redesigning the sterile logistics processes can increase effectiveness of hospitals, as it is widely sought.

With uncertainty of redesigning existing processes, simulation provides a cost-efficient approach to foresee feasibility of new solutions while material flow simulations [10] have been broadly employed in various fields of logistics.

The results and examples in this paper indicate that significant cost reductions are possible when making appropriate use of logistic principles, operations research methods, and IT.

\section{PROBLEM DEFINITION}

At the current state, the sterilization is performed in two separate facilities, where one is designated for sterilization of RMD of the many departments, while the facility within the central operation block manages sterilization of surgical instrument sets, which are sterilized directly after each performed surgery. Limited spatial and operations capacities of the surgery sterilization unit and separate sterilization facilities warrant for research into improving overall efficiency through introducing a central sterilization facility covering the sterilization process of the whole hospital. In the first phase, this requires understanding the requirements and performance of the current sterilization process and the material flow.

The daily RMD consumption of various departments is relatively constant and sterilized instruments can be provided on time at the beginning of the work shift, the anticipated impact on the new sterilization process is not time critical. Special attention, however, must be brought to managing sterilization of surgical instruments. Scheduled or unscheduled operations require sterile instrument sets on time. While the current sterilization organization manages to provide this, the sterilization workload often reaches maximum capacity, which requires speedy handling and can lead to increasing the probability of errors in a crucial hospital process. Redesigning a working process may lead to unanticipated pitfalls that could prevent normal operation. The aim of this paper is to provide means for analysing the feasibility of a redesigned process based on the expected workload.

At any point the RMDs can be in one of the following states:

- In use

- On transport (to or from sterilization)

- In sterilization

- In Storage.

To ensure an unobstructed process each sterile surgical instrument set needs to be available on time for each forthcoming surgery, which is done using simulation.

\section{CENTRAL STERILIZATION SIMULATION}

A two-stage simulation is employed in order to first analyse the performance characteristics of the central sterilization unit and afterwards determine the feasibility by integrating the central sterilization with other processes.

\subsection{Central Sterilization Model}

The sterilization system consists of three thermodisinfectors, three sterilizers and between 2 and 4 operators for manipulating the devices and RMDs. The material flow begins by loading the thermos-disinfectors, which is followed by unloading the thermos-disinfectors and loading the sterilizers, after which the sterile material is stored. The simulation model is based on capacities, specified operation times of the devices and estimated manipulation time of operators, which includes loading, unloading, and assembly of the material (see Fig. 1). The simulation model depends on the arrival rate $\lambda$ of RMDs which accumulate in the first queue before the three thermo-disinfectors with service rate $\mu_{\mathrm{T}}$ followed by another waiting queue and finally the last stage consisting of three sterilizers with two different specifications with service rates $\mu_{\mathrm{S} 1}$ and $\mu_{\mathrm{S} 2}$.

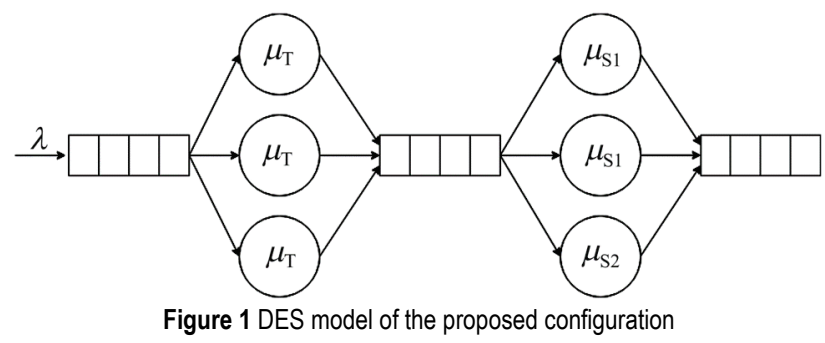

Table 1 Sterilization system specifications

\begin{tabular}{|l|c|c|c|c|}
\hline \multicolumn{1}{|c|}{ Device } & $\begin{array}{c}\text { Number } \\
\text { of }\end{array}$ & $\begin{array}{c}\text { Cycle- } \\
\text { time (min) }\end{array}$ & $\begin{array}{c}\text { Manipulation } \\
\text { time (min) }\end{array}$ & $\begin{array}{c}\text { Capacity } \\
\text { (pcs) }\end{array}$ \\
\hline Disinfector & 3 & 60 & 15 & 10 \\
\hline Sterilizers type 1 & 2 & 45 & 20 & 6 \\
\hline Sterilizer type 2 & 1 & 45 & 20 & 9 \\
\hline
\end{tabular}

The sterilization system model (Fig. 1) consists of servers representing the thermo-disinfectors and sterilizers and waiting queues in front of each server for accumulation of the material. The service rates for the servers can be expressed by the summed cycle and manipulation times with Eqs. (1)-(3). 


$$
\begin{aligned}
& \mu_{\mathrm{T}}=\frac{c_{t}}{t_{t}}=\frac{8}{h} \\
& \mu_{\mathrm{S} 1}=\mu_{\mathrm{S} 2}=\frac{c_{s 1}}{t_{s}}=\frac{5.53}{h} \\
& \mu_{\mathrm{S} 3}=\frac{c_{s 3}}{t_{s 3}}=\frac{8.30}{h}
\end{aligned}
$$

While the first level servers (thermo-disinfectors) technically provide the highest throughput, the overall performance depends heavily on the workload. With a maxed arrival intensity, the bottlenecks are caused by the sterilizers due to their limited capacity. With a workload below the cumulative capacity of the sterilizers, the bottleneck is caused by thermo-disinfectors due to their longer cycle times.

Discrete event simulation (DES) the maximum system output at maximal input load reaches 18 sterile surgical instrument sets per hour, while a complete cycle from the beginning to the end of sterilization for a single instrument set adds up to 140 minutes.

\subsection{Central Sterilization Workload Data}

The throughput of the central sterilization depends on the actual workload. While the sterilization of all of the hospital's material may require two or more shifts the bulk of the expected load does not require time crucial handling. However, the sterilization of surgical instrument sets must be performed on time in order to prevent omission of planned surgeries. To anticipate the workload, surgeries performed in the period of 3 months were observed. For this purpose, a list of all available surgical instrument sets was first made. Altogether the list contains slightly above 300 surgical instrument sets. Depending on the surgery type there may be several equivalent instrument sets available or they are unique for less frequent surgeries. Tab. 2 shows a sample of more frequently used instrument sets.

Table 2 Example of some most used instrument sets.

\begin{tabular}{|c|l|l|}
\hline ID & \multicolumn{1}{|c|}{ Set name } & \multicolumn{1}{|c|}{ Srugery type } \\
\hline 201 & ABDOMEN 1 & ABDOMINAL \\
\hline 202 & ABDOMEN 2 & ABDOMINAL \\
\hline 205 & LAP OLD & ABDOMINAL \\
\hline 208 & LAPAROSCOPE 0? & ABDOMINAL \\
\hline 210 & HOLDER NORMAL & ABDOMINAL \\
\hline 211 & STAPLER 1 & ABDOMINAL \\
\hline 300 & LARGE COLIBRI & TRAUMA \\
\hline 301 & LARGE BONE & TRAUMA \\
\hline 305 & TEP-PEP & TRAUMA \\
\hline 322 & APTUS & TRAUMA \\
\hline 323 & AESCULAP OSCILACTION SAW & TRAUMA \\
\hline 355 & LIGAMENT SET 1 & TRAUMA \\
\hline 401 & ABRASIO 1 & VEINS \\
\hline 406 & CONISATION & VEINS \\
\hline 410 & HSG 1 & VEINS \\
\hline 418 & LPSC 1 & VEINS \\
\hline 433 & VLSC & VEINS \\
\hline 701 & OZIL 1 $(4 x)$ & EYE \\
\hline 704 & EYELID PLASTICS $(4 \mathrm{x})$ & EYE \\
\hline
\end{tabular}

With the given instrument set list, about 2000 surgeries performed in three consecutive months were observed between September and including November of 2016, which represents the busiest time of the work year providing the necessary conditions for analysis. For each of the performed surgeries a list of required surgical instrument sets was defined, which includes alternative combinations where missing instrument sets could be replaced with similar ones. Using the historic data, the workload of the previously performed operations was defined. While this only counts for the material usage of performed surgeries and not for other medical procedures, they represent the time-critical part of the sterilization system.

The used data about the performed surgeries include:

- $\quad$ Surgery type

- Surgery start

- Surgery duration

- Operating room

- List of used instrument sets or their alternatives.

For the analysis of the instrument set arrival intensity any of the combinations might be feasible, however to determine the usage of the instrument sets only one of the combinations was used for each surgery. The historical arrival intensity of surgical instrument sets of performed surgeries can be seen in Fig. 2.

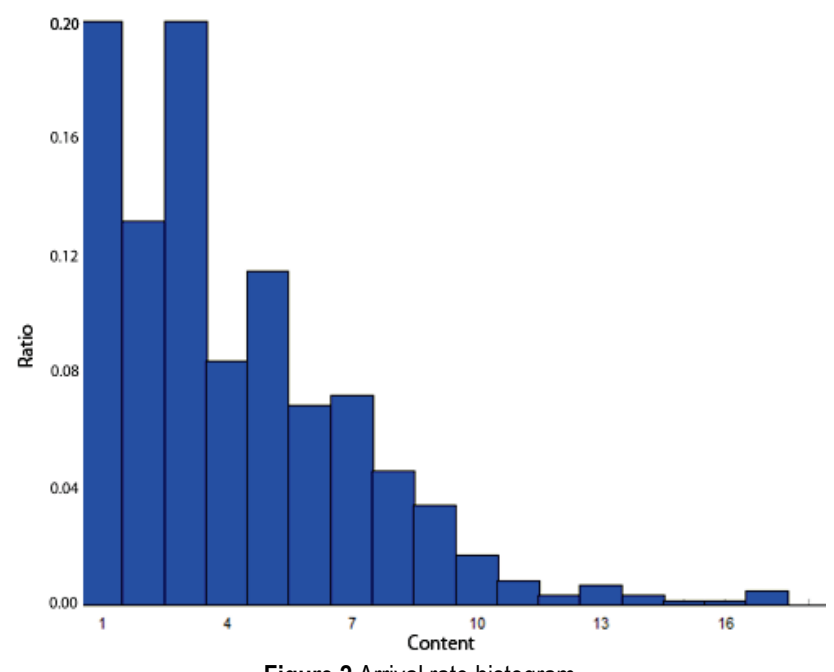

Figure 2 Arrival rate histogram

According to the specification of the central sterilization there are only a few cases in which the arrival intensity reaches the system capacity. In most cases only one or two processing lines are used (not regarding sterilization of other medical equipment). Regarding the capacity and performance of the central sterilization unit can follow the requirements. However, given that the number of instrument sets intended for specific surgeries is limited, this simulation result is not yet sufficient to guarantee timely supply of instruments for each planned surgery.

\subsection{Simulation of the Logistics Process}

To simulate the whole process that covers the sterilization as well as supply of both sterilization and operating theatres it is necessary to consider any delays that may appear in the process. Delays that appear include:

- Waiting for transportation after surgery

- Transport times 
- Waiting for transportation after sterilization

- Delays due to reached sterilization capacity.

Deliveries to central sterilization and the operating block are not done directly after surgeries, instead they are scheduled hourly for simulation purposes. Pessimistic estimation of transportation times was determined as 15 minutes after measuring the time on transportation routes. The process follows use of sterile instrument sets on surgery, transportation to sterilization, sterilization, and finally transportation and storage of sterile material.

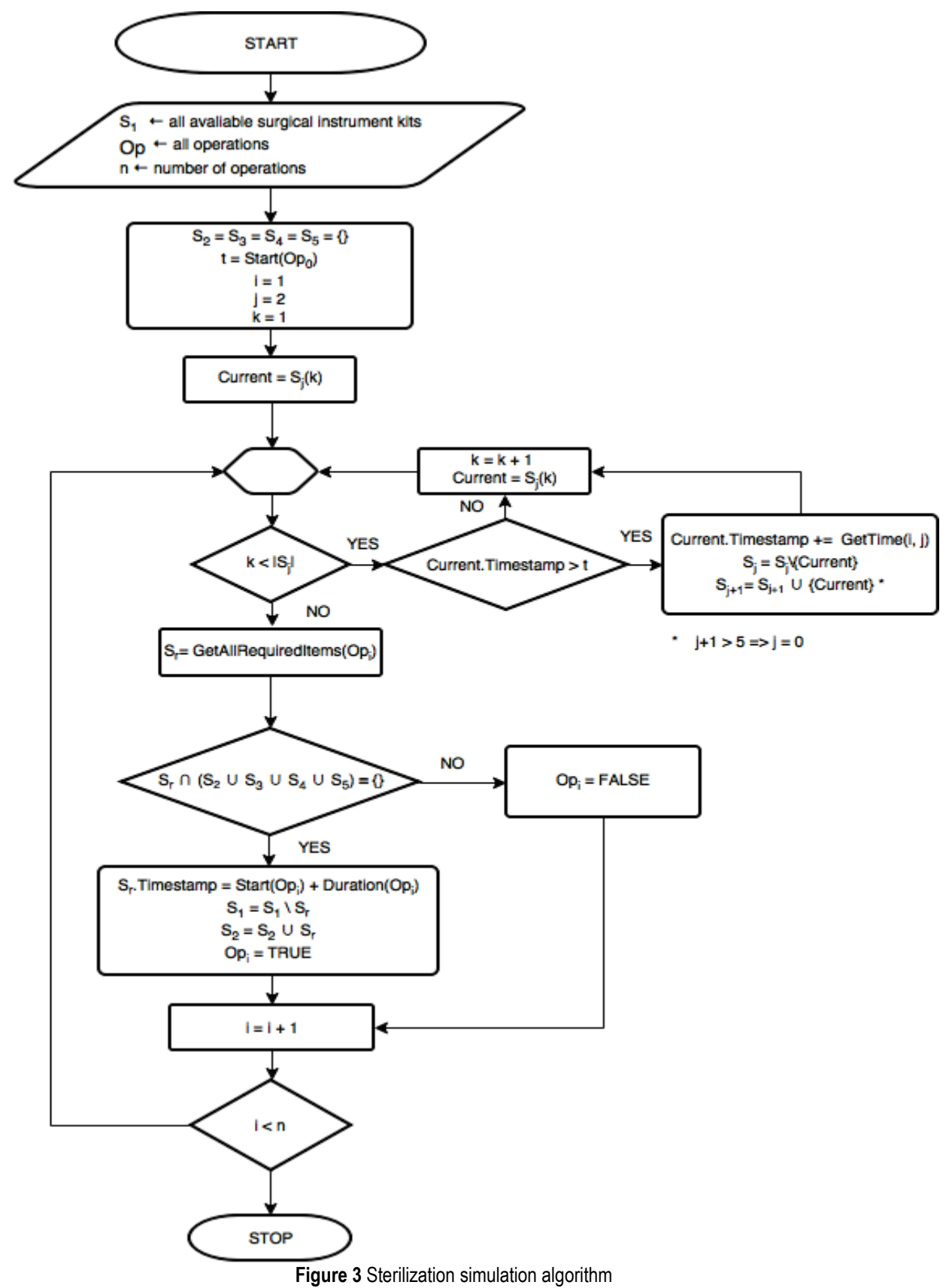

With the bulk of surgeries being performed in the morning and early afternoon, sterilization of other medical equipment can be postponed to later hours and be ready for use the next day. The surgical instrument sets however need to be available when required. Using the limited stock of surgical instrument sets the surgery requirements were tested for availability. By simulation instrument set consumption on surgeries, transportation, and sterilization.

For this purpose, all operations were first sorted by time of beginning. For each operation the available instrument sets were checked for availability. In case all required was available, they were assigned a timestamp based on the start of the surgery and its duration and removed from the availability list and moved into the list of instrument sets in use. If all required instrument sets are not found, then the surgery is not performed. Following this, the timestamp for each instrument set in use is checked whether its timestamp exceeds the time of the current operation start. In this case the timestamp is increased to the scheduled time for transportation to sterilization and transportation time and the instrument sets are transferred from the list in use to the transportation list. The same check is done with the instrument sets in the transportation list, which increases the timestamp to the 
scheduled sterilization finish and moves the instrument sets into the sterilization list. The next check is done for the sterilization list which again readjusts the timestamp for transportation to storage. The last list is checked whether any instrument sets exceeds the current time, in which case it resets its timestamp to 0 and moves those instrument sets into available list. The flowchart in Fig. 3 depicts the simulation algorithm.

\section{RESULTS AND DISCUSSION}

Each of the instrument sets was linked to the data of performed surgeries. Tab. 3 shows the analysis of available instrument sets for scheduled surgeries in typical morning workday.

\begin{tabular}{|c|c|c|c|c|}
\hline Table 3 Simulation of surgery feasibility \\
\hline Date & Start & $\begin{array}{c}\text { Duration } \\
(\mathrm{m})\end{array}$ & Required set IDs & $\begin{array}{c}\text { Surgery } \\
\text { possible }\end{array}$ \\
\hline 1.09 .2016 & $08: 00: 00$ & 30 & 701 & Yes \\
\hline 1.09 .2016 & $08: 00: 00$ & 55 & $433,410,418$ & Yes \\
\hline 1.09 .2016 & $08: 00: 00$ & 70 & $355,300,322$ & Yes \\
\hline 1.09 .2016 & $09: 00: 00$ & 25 & 701 & Yes \\
\hline 1.09 .2016 & $10: 00: 00$ & 120 & $201,211,210$ & Yes \\
\hline 1.09 .2016 & $10: 00: 00$ & 90 & $301,323,305,306$ & Yes \\
\hline 1.09 .2016 & $10: 00: 00$ & 30 & 406 & Yes \\
\hline 1.09 .2016 & $11: 00: 00$ & $/$ & 433 & No \\
\hline 1.09 .2016 & $12: 00: 00$ & 79 & 205,208 & Yes \\
\hline 1.09 .2016 & $12: 00: 00$ & 30 & 216 & Yes \\
\hline 1.09 .2016 & $12: 00: 00$ & $/$ & 322 & No \\
\hline 1.09 .2016 & $12: 00: 00$ & 15 & 401 & Yes \\
\hline 1.09 .2016 & $13: 00: 00$ & $/$ & 205 & No \\
\hline 1.09 .2016 & $14: 00: 00$ & $/$ & 216 & No \\
\hline 1.09 .2016 & $14: 00: 00$ & 10 & 704 & Yes \\
\hline 1.09 .2016 & $14: 00: 00$ & 10 & 704 & Yes \\
\hline 1.09 .2016 & $14: 00: 00$ & 10 & 704 & Yes \\
\hline 1.09 .2016 & $14: 00: 00$ & $/$ & $305,306,307,323$ & No \\
\hline
\end{tabular}

Any instrument set that cannot be made available after the end of surgery, sterilization, and transport causes the cancelation of the planned surgery. The instrument sets are made available for the next surgery after finishing the sterilization and transport.

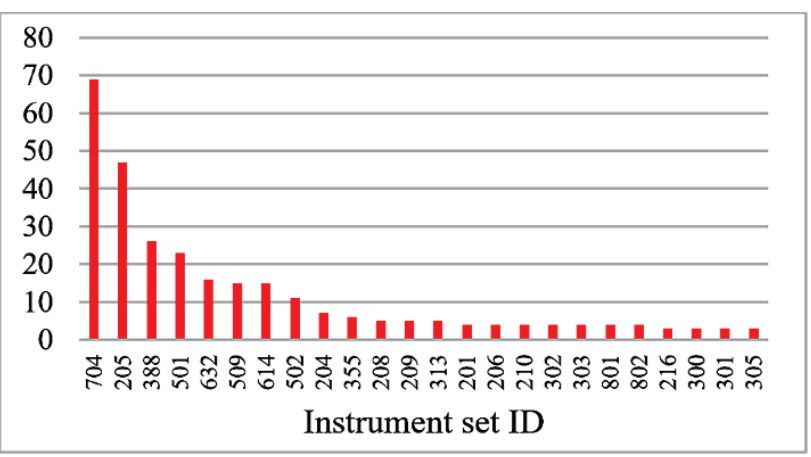

Figure 4 Frequency of simulated missing surgical instrument sets

Analysis of the performed surgeries revealed insufficient sterile surgical instrument sets for $12 \%$ of scheduled surgeries. Occasional surgeries would be cancelled due to a single missing instrument set, however most often whole combinations of required instrument sets that are required for surgeries of the same type would be missing. This is more obvious for trauma and vascular surgeries that require a broader combination of instrument sets. The simulation also revealed missing instrument sets
(Fig. 4) causing cancelled surgeries, which are interestingly not entirely correlated with the frequency of instrument set usage (Fig. 5), implying that use frequency is not the reason for failure. Both figures show the most frequently used or missed instrument sets.

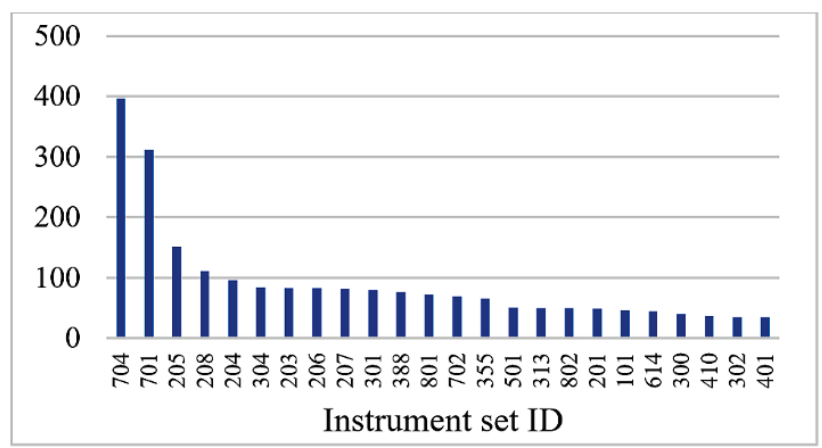

Figure 5 Frequency of used surgical instrument sets in the 3-month period

The cause for failing to meet about $12 \%$ of surgeries lies in both the longer cycle times for instrument sets to become available and low count for specific instrument sets, which becomes most apparent for eye surgeries. While eye surgeries are most frequent, two operation types dominate with the same frequency and same number of available surgical instrument sets. However, the simulation revealed that the one operation type (that requires instrument set 704) causes failure to meet the schedule (above 60 failures), while the impact of the required set 701 is minimal. This indicates that the cause of missed surgeries is not entirely caused by low count of instrument sets for specific surgeries, but instead due to their scheduling, meaning that the planned central sterilization would not be able to completely satisfy the requirements of surgeries as they were performed.

\section{CONCLUSION}

Alternative scenarios were tested in order to determine whether the surgeries could be performed completely under other circumstances. For this purpose, acquisition of additional surgical instrument sets was anticipated. The simulation results revealed that doubling or in some cases (the first 8 sets given in Fig. 4) tripling the amount of missing instrument sets increases the success of surgery schedule to $98 \%$. In this instance the surgeries in table 2 would have been all possible. However, to completely fulfil it certain instrument sets require an even higher stock. As the cost of acquiring new surgical equipment is not insignificant another solution may be more viable.

As mentioned earlier, the variation between the frequency of used and missing instrument sets suggests that scheduling may be a more important factor. For this purpose, the surgery schedule was altered in order to provide test the central sterilization with the same workload but at different times. Consecutive surgeries of similar type, which is the main reason for missing instrument sets were rescheduled to later times or swapped with surgeries of different type with similar duration. Using this straightforward rescheduling improved the percentage of successfully scheduled surgeries to $97 \%$, while combining both methods (with a slight stock increase) allowed for completely fulfilled schedule. 
At the end, we can claim that the studied problem can be solved by two different strategies. First strategy is an acquisition of additional surgical instrument, while the second one is careful planning and automated surgery scheduling. The automated surgery scheduling is a promising topic for the future research, which will probably reveal that the combination of both strategies is the best, while the method presented in this paper allows for testing the feasibility of scheduling efficiency. Future work will focus on comparing both strategies from a cost view, providing optimization through simulation in order to find the most cost effective solution.

\section{REFERENCES}

[1] Stebbins, S. \& Frohlich, T. C. (2015). Countries spending the most on health care. Available: www.usatoday.com/story/ money/business/2015/11/14/24-7-wall-st-countries-spendmost-health-care/75771044/. (Accessed 27 March 2018).

[2] van de Klundert, J., Muls, P., \& Schadd, M. (2008). Optimizing sterilization logistics in hospitals. Health Care Management Science, 23-33. https://doi.org/10.1007/s10729-007-9037-4

[3] Carter, M. (2002). Diagnosis: mismanagement of resources. OR/MS Today, 29(2).

[4] Dutch Ministry of Health. (2003). Annual report.

[5] Reymondon, F., Pellet, B., \& Marcon, E. (2008). Optimization of hospital sterilization costs proposing new grouping choices of medical devices into packages. International Journal of Production Economics, 112, 326335. https://doi.org/10.1016/j.ijpe.2006.12.066

[6] Tlahig, H., Jebali, A., Bouchriha, H., \& Ladet, P. (2013). Centralized versus distributed sterilization service: A location-allocation decision model. Operations Research for Health Care, 2, 75-85. https://doi.org/10.1016/j.orhc.2013.05.001

[7] Tlahig, H., Jebali, A., Bouchriha, H., \& Ladet, P. (2009). A two-phased approach for the centralization vs. decentralization of the hospital sterilization department. European Journal of Industrial Engineering, 227-246. https://doi.org/10.1504/EJIE.2009.023606

[8] Ozturk, O., Espinouse, M., Di Mascolo, M., \& Gouin, A. (2010). Optimizing the makespan of washing operations of medical devices in hospital sterilization services. Proceedings of IEEE Workshop on Health Care Management (WHCM), Venice. https://doi.org/10.1109/WHCM.2010.5441278

[9] Nikoličić, S., Kilibarda, M., Atanasković, P., Duđak, L., \& Ivanišević, A. (2015). Impact of RFID Technology on Logistic Process Efficiency in Retail Supply Chains. PROMET - Traffic \& Transportation, 27(2), 137-146. https://doi.org/10.7307/ptt.v27i2.1539

[10] Dvornik, J., Munitic, A., \& Bilic, M. (2006). Simulation modelling and heuristics optimization of material flow of the port cargo system. PROMET - Traffic \& Transportation, $18(2), 123-135$.

[11] Syama, S. S. \& Côté, M. J. (2012). A comprehensive location-allocation method for specialized healthcare services. Operations Research for Health Care, 1(4), 73-83. https://doi.org/10.1016/j.orhc.2012.09.001

[12] Silver, E. A., Pyke, D. E., \& Peterson, R. (1998). Inventory management and production planning and scheduling. Wiley.

\section{Contact information:}

Bojan RUPNIK, PhD

(Corresponding author)

University of Maribor, Faculty of Logistics, Mariborska cesta 7, 3000 Celje, Slovenia

bojan.rupnik@um.s

Radivoj NARDING, MSc

General hospital Izola,

Polje 40, 6310 Izola, Slovenia

radivoj.nardin@sb-Izola.si

Tomaž KRAMBERGER, PhD

University of Maribor, Faculty of Logistics, Mariborska cesta 7, 3000 Celje, Slovenia tomaz.kramberger@um.si 